\title{
MINIMIZAÇÃO DO PESO DE TRELIÇAS DE ALUMÍNIO PELA OTIMIZAÇÃO SIMULTÂNEA DA SEÇÃO TRANSVERSAL DOS ELEMENTOS E DA GEOMETRIA: ANÁLISE NUMÉRICA E VALIDAÇÃO EXPERIMENTAL
}

\section{Minimization of Truss Weigth by Simultaneous Optimization of Cross- Sectional Dimensions and Geometry: Numerical Analysis and Experimental Validation}

\author{
Moacir Kripka ${ }^{1}$, Zacarias M. Chamberlain Pravia ${ }^{2}$, \\ Maiga Marques Dias ${ }^{3}$, Guilherme Fleith de Medeiros ${ }^{4}$
}

Recebido em 29 de setembro de 2013; recebido para revisão em 21 de outubro de 2013; aceito em 15 de novembro de 2013; disponivel on-line em 21 de novembro de 2013.

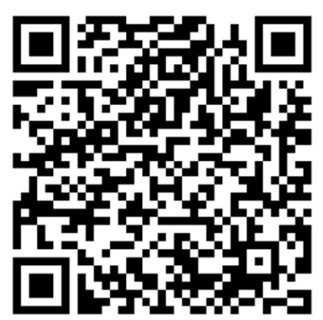

PALAVRAS CHAVE:

Treliças de alumínio, Análise numérica, Análise experimental;

\section{KEYWORDS:}

Aluminum trusses, Numerical analysis, Experimental analysis

\begin{abstract}
RESUMO: Em função da grande diversidade de configurações possíveis, as treliças constituem um campo bastante fértil ao emprego de técnicals de otimização. Com o uso dessas técnicas, busca-se quantificar o mínimo material necessário para que a estrutura suporte o carregamento atuante com a devida segurança. Os problemas de otimização de treliças são classificados basicamente em três categorias: otimização de seções transversais, de geometria e de topologia. O presente trabalho apresenta um estudo numérico e experimental desenvolvido na Universidade de Passo Fundo, através do qual objetivou-se a determinação de parâmetros para o dimensionamento ótimo de treliças considerando tanto as seções transversais dos elementos como a geometria como variáveis de projeto. Inicialmente, foram efetuadas diversas simulações com o emprego de um programa desenvolvido para a otimização de treliças metálicas pelo método Simulated Annealing. Na sequência, a partir dos resultados obtidos, foram projetadas e construídas duas estruturas treliçadas de alumínio. Os ensaios efetuados evidenciaram a significativa melhora no desempenho da estrutura projetada com o emprego das técnicas de otimização.
\end{abstract}

ABSTRACT: Due to the wide variety of possible configurations, the trusses consist in a rich field to the application of optimization techniques. Using these techniques, we seek to quantify the minimum material needed to support the applied loads safely. The optimization problems of trusses are classified into three basic categories: optimization of cross sections, geometry and topology. This paper presents a numerical and experimental study developed in order to propose parameters for the optimum design of trusses considering both the cross sections of elements and the geometry as design variables. Initially, several numerical simulations were performed with the use of a software developed by the association of a dimensioning routine with the Simulated Annealing Method. In the sequence, from the results obtained, we designed and built two aluminum truss structures. The tests performed showed a significant improvement in the performance of the structure designed with the aid of optimization techniques.

\footnotetext{
* Contato com os autores:

${ }^{1}$ e-mail : mkripka@upf.br (M. Kripka)

Professor Doutor, Universidade de Passo Fundo, RS, Brasil, Programa de Pós-Graduação em Engenhariia Civil e Ambiental

22e-mail : zacarias@upf.br ( Z. M. C. Pravia)

Professor Doutor, Universidade de Passo Fundo, RS, Brasil, Programa de Pós-Graduação em Engenhariia Civil e Ambiental

${ }^{3}$ e-mail : maiga@upf.br (M. M. Dias)

Professora Mestre, Universidade de Passo Fundo, RS, Brasil, curso de Engenharia Civil

${ }^{4}$ e-mail : guifleith@upf.br ( G. F. de Medeiros )

Professor Mestre, Universidade de Passo Fundo, RS, Brasil, curso de Engenharia Civil
} 


\section{INTRODUÇÃO}

As treliças possuem um vasto campo de aplicação na engenharia, sendo muito utilizadas na construção de pontes (rodoviárias e ferroviárias), como estruturas de cobertura (em residências, indústrias, estádios, etc), em torres de transmissão de energia, entre diversos outros usos. São construídas usualmente em madeira ou aço, sendo relativamente leves e especialmente indicadas para superar grandes vãos ou suportar cargas elevadas. Nesse contexto, as treliças tornam-se uma solução econômica e prática. Além disso, devido à grande diversidade de soluções estaticamente possíveis para se vencer um determinado vão ou suportar um determinado carregamento, as treliças apresentam um campo bastante fértil ao emprego de técnicas de otimização.

Pelo fato da análise e dimensionamento de estruturas, de forma geral, se constituírem em processos iterativos, busca-se, com o uso dessas técnicas, minimizar o consumo de material sem o comprometimento da segurança estrutural. Os problemas de otimização de treliças são dispostos basicamente em três categorias: otimização das seções transversais, da geometria e da topologia. Na primeira situação, as variáveis consideradas no projeto são apenas as características das seções transversais das barras. Neste caso, pode-se trabalhar com variáveis discretas, através de um conjunto de dimensões pré-definidas para as seções, ou variáveis contínuas, quando são aceitos quaisquer valores de seção dentro de um determinado intervalo. Na otimização da geometria permite-se a modificação das coordenadas dos nós para uma topologia pré-definida. Nessa situação também é comum facultar-se a modificação das características das seções transversais. No caso da otimização da topologia, tanto o número como a disposição dos elementos na estrutura podem variar. O processo de otimização de treliças pode apresentar uma ou mais das seguintes restrições básicas: deslocamento admissível (flecha), tensão máxima de flambagem e tensão axial máxima.

O presente trabalho objetivou a validação de estudos numéricos envolvendo a otimização simultânea de seção transversal e de geometria de treliças de alumínio. Inicialmente, foram efetuadas simulações computacionais com o emprego de um programa desenvolvido para a otimização de treliças planas pelo Método Simulated Annealing. A seguir, foram construídas e ensaiadas duas treliças de alumínio, dimensionadas para suportar o mesmo carregamento. Para o primeiro modelo construído foi efetuada apenas a otimização de seções, enquanto para o segundo permitiu-se a inclusão das coordenadas dos nós como variáveis de projeto, otimizando de forma simultânea a geometria da estrutura e as seções transversais dos elementos.

Os itens seguintes deste trabalho descrevem a metodologia empregada no estudo, bem como os resultados e conclusões decorrentes.

\section{OTIMIZAÇÃO DE TRELIÇAS}

\subsection{SISTEMA COMPUTACIONAL PARA OTIMIZAÇÃO DE TRELIÇAS}

Um problema de otimização pode, de forma geral, ser descrito conforme Equação 01:

Minimizar:

$$
f\left(x_{i}\right) \text {, sendo } i=1, n
$$

Sujeito a:

$$
\begin{aligned}
& g_{j}\left(x_{i}\right) \leq 0, \text { sendo } j=1, m \\
& h_{k}\left(x_{i}\right)=0, \text { sendo } k=1, l \\
& x_{i}^{l} \leq x_{i} \leq x_{i}^{u}
\end{aligned}
$$

Onde $f$ designa a função objetivo e $X=\left(x_{1}\right.$, $\left.x_{2}, \ldots x_{n}\right)^{T}$ consiste no vetor das variáveis de projeto. As demais funções são as chamadas restrições do problema (respectivamente, restrições de desigualdade $g$, de igualdade $h$ e restrições laterais, ou canalizadas, com limite inferior / e limite superior $u$ ). As funções envolvidas no problema podem conter as variáveis de projeto de forma implícita ou explícita. Além disso, tanto a função objetivo como as restrições podem ser lineares ou não-lineares.

As diversas técnicas de programação 
matemática para otimização de estruturas possuem grandes limitações no que diz respeito a suas aplicações em problemas práticos, devido ao fato das funções necessitarem apresentar características como convexidade e continuidade (tanto da função como de suas derivadas). As funções que envolvem o cálculo de estruturas, ao contrário, usualmente são descontínuas e nãoconvexas (apresentando vários pontos de ótimo). Para problemas dessa natureza, as heurísticas têm se apresentado como uma excelente alternativa.

O processo de otimização de treliças descrito na sequência foi efetuado utilizando-se um sistema computacional desenvolvido pelo primeiro autor deste trabalho, o qual associa a análise de estruturas reticuladas pelo Método dos Deslocamentos com o Método Simulated Annealing (KIRKPATRICK et al., 1983). O Simulated Annealing consiste num método heurístico desenvolvido em analogia ao processo de recozimento de metais, e vêm sendo empregado com excelentes resultados pelos autores. Uma descrição mais detalhada do método, bem como de sua aplicação específica à otimização de treliças, pode ser encontrada em Kripka (2004) ou em Kripka e Drehmer (2005).

$\mathrm{Na}$ implementação do sistema, o problema de minimização do peso de treliças foi formulado da seguinte forma:

Minimizar:

$$
f(x)=W=\sum_{i=1}^{n} \gamma A_{i} L_{i}
$$

Sujeito a:

$$
\begin{aligned}
& \sigma_{\mathrm{i}} \leq \sigma_{\mathrm{a}} \\
& \lambda \leq \lambda_{\lim } \\
& u_{\mathrm{i}} \leq u_{\mathrm{a}} \\
& A \in S=\left\{a_{1}, \ldots a_{m}\right\} \\
& x^{l} \leq x_{i} \leq x^{u}
\end{aligned}
$$

Na função objetivo, $W$ é o peso total da treliça, o qual é função da área da seção transversal $(A)$ e das coordenadas dos nós da treliça $(x)$. Ainda nessa função, $\gamma$ é o peso específico do material e $L$ é o comprimento do elemento, sendo $n$ o número total de elementos. Em cada elemento tem-se como restrição que a tensão $\sigma_{i}$ em cada barra deve ser menor ou igual à tensão admissível do material $\sigma_{a}$, sendo considerado ainda o índice de esbeltez máximo $\left(\lambda_{\text {lim }}\right)$ para os elementos comprimidos e um deslocamento admissível $u_{a}$ para cada nó da estrutura.

Além das restrições descritas, considerouse que a área da seção deve assumir um valor discreto, a partir de uma lista de $m$ perfis candidatos (equação 9). Por fim, a última restrição, representada na equação 10 , impõe limites para a variação nas coordenadas de cada nó.

\subsection{OTIMIZAÇÃO APLICADA AO DIMENSIONAMENTO DE TRELIÇAS PLANAS DE ALUMÍNIO}

Com o objetivo de efetuar a comprovação da efetiva economia obtida a partir da aplicação de técnicas de otimização à análise de estruturas, o sistema computacional anteriormente desenvolvido foi adaptado à otimização de treliças compostas por perfis de alumínio. Uma vez que a disponibilidade de distintos perfis era bastante limitada, visou-se predominantemente a minimização do peso da estrutura pela alteração nas coordenadas nodais.

O material utilizado foi o alumínio liga 6351, têmpera T4, com as seguintes características (BUZINELLI, 2000):

- módulo de elasticidade longitudinal E = 70 GPa;

- peso específico $\gamma=27 \mathrm{KN} / \mathrm{m}^{3}$;

- tensão de escoamento fy = $130 \mathrm{MPa}$;

- tensão última fu = $220 \mathrm{MPa}$;

- índice de esbeltez máximo $\lambda_{\lim }=111$.

Foram utilizados três tipos de perfis laminados em forma de dupla cantoneira de abas iguais e opostas, sendo suas características expostas na Tabela 1 (ALCOA, 2013).

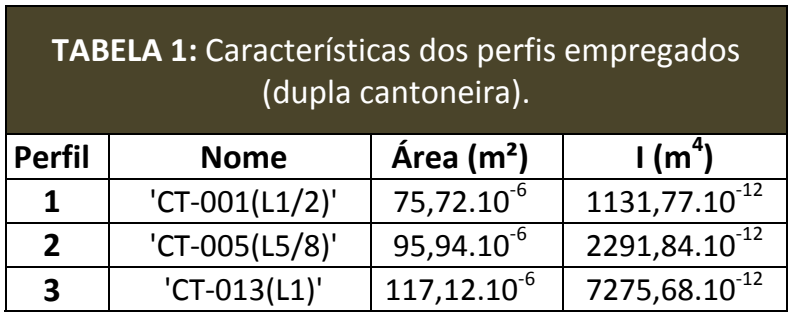


Foi adotado o mesmo valor de tensão admissível tanto para a compressão como para a tração, sendo os coeficientes de segurança iguais a 1,65 para a tensão de escoamento e 2 para a tensão última e, consequentemente, a tensão admissível o menor valor dentre os seguintes, Equações 11 e 12:

$$
\begin{gathered}
\sigma_{a d m}=\frac{f y}{1,65}=\frac{130}{1,65}=78,79 \mathrm{MPa} \\
\sigma_{a d m}=\frac{f u}{2,00}=\frac{220}{2,00}=110 \mathrm{MPa}
\end{gathered}
$$

A tensão de flambagem é calculada a partir da Equação 13:

$$
\sigma_{f l}=\frac{145-0,8 \lambda}{1,65}
$$

\section{SIMULAÇÕES NUMÉRICAS}

A estrutura de referência empregada no presente estudo consiste numa treliça plana de banzos paralelos do tipo Warren, com $1,75 \mathrm{~m}$ de vão e $0,25 \mathrm{~m}$ de altura (Figura 1). O carregamento, aplicado no nó central do banzo superior, foi determinado como a maior carga suportada pela estrutura para todos os elementos dimensionados com o perfil do tipo 2 (Tabela 1 ), e que resultou em $3,2 \mathrm{KN}$.

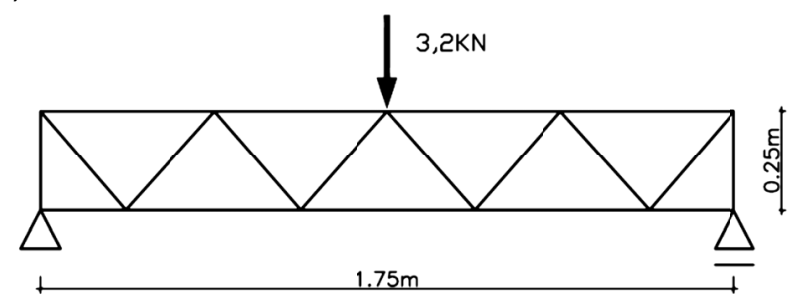

Figura 1: Estrutura de referência.

Partindo da estrutura de referência foram sendo efetuadas diversas simulações, para as quais foram sendo definidas distintas restrições e variáveis de projeto. A exemplo das seções transversais, as coordenadas nodais foram consideradas como variáveis discretas (múltiplos de um centímetro). Em todas essas simulações, tanto as coordenadas dos nós dos apoios como a coordenada horizontal do nó de aplicação do carregamento foram mantidas fixas. Foram consideradas as seguintes variáveis e restrições, listadas em ordem crescente de complexidade:

1a. Variáveis: seções transversais dos elementos, divididos em três grupos: banzo superior, banzo inferior e demais elementos (diagonais e montantesi); posições dos nós fixas;

2a. Todas as coordenadas verticais móveis e um único valor de seção para todos os elementos;

3a. Coordenadas verticais do banzo superior móveis e três grupos de elementos (banzo superior, banzo inferior e demais elementos) variando a seção de forma independente;

4a. Todas as coordenadas verticais móveis e três grupos de elementos (banzo superior, banzo inferior e demais elementos) variando a seção de forma independente;

5a. Coordenadas verticais do banzo superior e coordenadas horizontais do banzo inferior móveis e três grupos de elementos (banzo superior, banzo inferior e demais elementos) variando a seção de forma independente;

6a. Coordenadas verticais e horizontais do banzo superior e coordenadas horizontais do banzo inferior móveis, além de três grupos de elementos (banzo superior, banzo inferior e demais elementos) variando a seção de forma independente;

7ạ. Todas coordenadas verticais e horizontais móveis e três grupos de elementos (banzo superior, banzo inferior e demais elementos) variando a seção de forma independente;

Cabe observar que, em função da pouca diversidade de perfis, a siituação correspondente à primeira simulação é de solução bastante simples, sendo no caso de caráter apenas ilustrativo. No entanto, na medida em que passa-se a considerar um número maior de variáveis de projeto, o número de combinações possíveis cresce de forma não polinomial, aumentando de forma considerável a complexidade do problema e, consequentemente, o ganho relativo obtido com o emprego da otimização. 
Através das simulações efetuadas pode-se perceber claramente a maior variação percentual obtida no consumo de material à medida que se aumentou o número de variáveis de projeto e, consequentemente, a complexidade do processo de otimização. Na Tabela 2 são apresentadas as porcentagens de variação de peso para cada uma das situações anteriormente descritas, em relação à estrutura de referência. Na Figura 2 apresentam-se os modelos correspondentes gerados pelo software de otimização, sendo que o perfil 1 é representado pela cor verde, e o perfil 2 pela cor vermelha. Nenhum modelo utilizou o perfil 3.

\begin{tabular}{|c|c|c|c|}
\hline \multicolumn{4}{|c|}{ TABELA 2: Resultados das simulações numéricas. } \\
\hline & Peso Inicial (KN) & Peso Final (KN) & Redução (\%) \\
\hline $\mathbf{1 a}$ & 0,01725 & 0,01552 & $10,00 \%$ \\
\hline $\mathbf{2} \mathbf{a}$ & 0,01725 & 0,01515 & $12,18 \%$ \\
\hline $\mathbf{3 a}$ & 0,01725 & 0,01430 & $17,09 \%$ \\
\hline $\mathbf{4 a}$ & 0,01725 & 0,01390 & $19,40 \%$ \\
\hline $\mathbf{5 a}$ & 0,01725 & 0,01343 & $22,16 \%$ \\
\hline $\mathbf{6} \mathbf{a}$ & 0,01725 & 0,01189 & $31,08 \%$ \\
\hline $\mathbf{7 a}$ & 0,01725 & 0,01169 & $32,25 \%$ \\
\hline
\end{tabular}

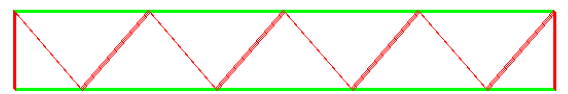

1a Simulação

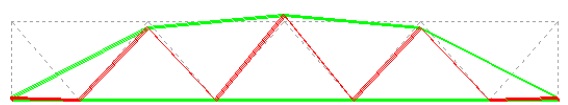

3a Simulação

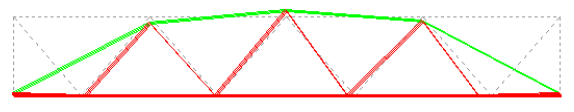

5a Simulação

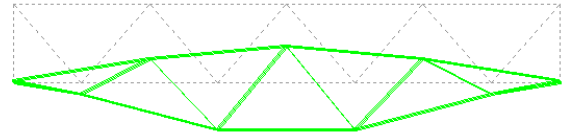

2a Simulação

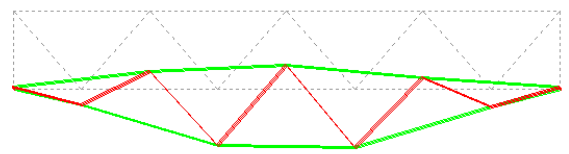

4ำ Simulação

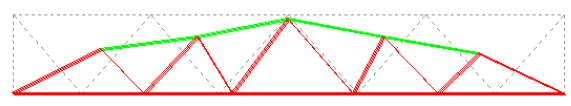

6a Simulação

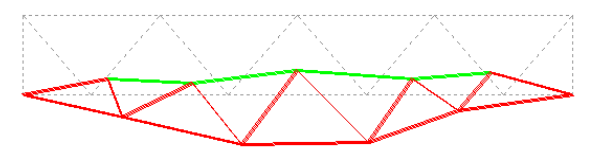

7a Simulação

Figura 2: Modelos resultantes das simulações. 


\section{ANÁLISE EXPERIMENTAL}

Para a construção dos dois modelos optou-se pelas treliças geradas na 1 a e na 6 a simulações, e que serão doravante designadas por Modelo 1 e Modelo 2, respectivamente. Apesar da 7ạ simulação ter resultado no menor consumo final de material, o ensaio do modelo correspondente traria maiores dificuldades devido à disposição dos elementos situados abaixo da linha dos apoios.

No Modelo 1 as seções dos banzos inferiores e superiores utilizaram o perfil 2, enquanto as demais barras foram construídas com o perfil 1 (Figura 3). Já no Modelo 2, apenas parte os elementos do banzo superior utilizaram o perfil 2 , sendo as demais barras formadas a partir do perfil 1 (Figura 4), resultado da maior uniformização no valor dos esforços solicitantes decorrente dos deslocamentos dos nós. Desta forma, apesar de ambos os modelos terem sido dimensionados para um mesmo carregamento $(3,2 \mathrm{kN})$, o Modelo 2 apresenta uma redução de aproximadamente 23,4 \% no peso com relação ao Modelo 1.

A Figura 5 apresenta os modelos construídos a partir dos resultados das simulações numéricas. Ambos os modelos foram ensaiados sob as mesmas condições de vinculação e de carregamento. Já a Figura 6 ilustra o Modelo 1 pronto para a aplicação da carga, enquanto a Figura 7, correspondente ao Modelo 2, pode-se observar com maior detalhe o travamento dos nós do banzo superior. Os resultados obtidos dos ensaios são apresentados de forma resumida na Tabela 3, na qual o peso próprio corresponde ao das estruturas efetivamente construídas.

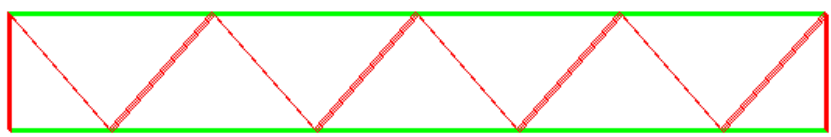

Figura 3: Modelo 1 gerado pelo software de otimização.

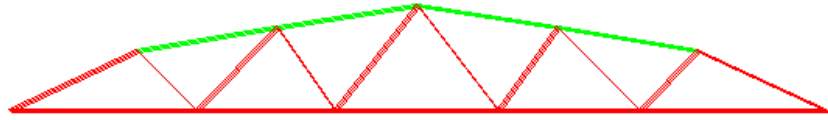

Figura 4: Modelo 2 gerado pelo software de otimização.

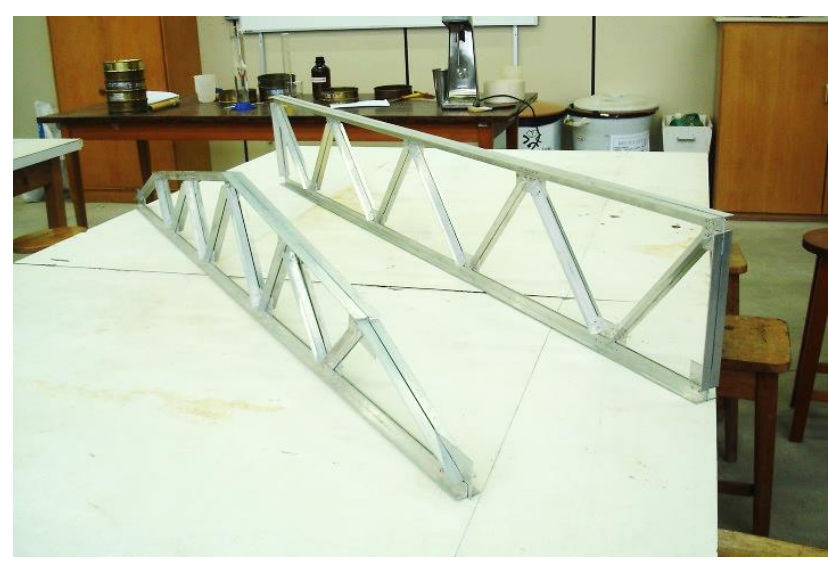

Figura 5: Modelos construídos.

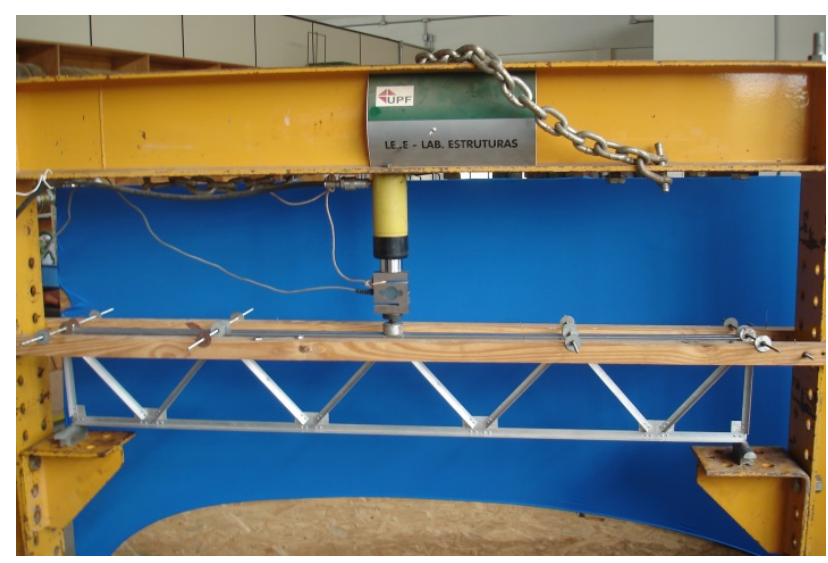

Figura 6: Modelo 1. 


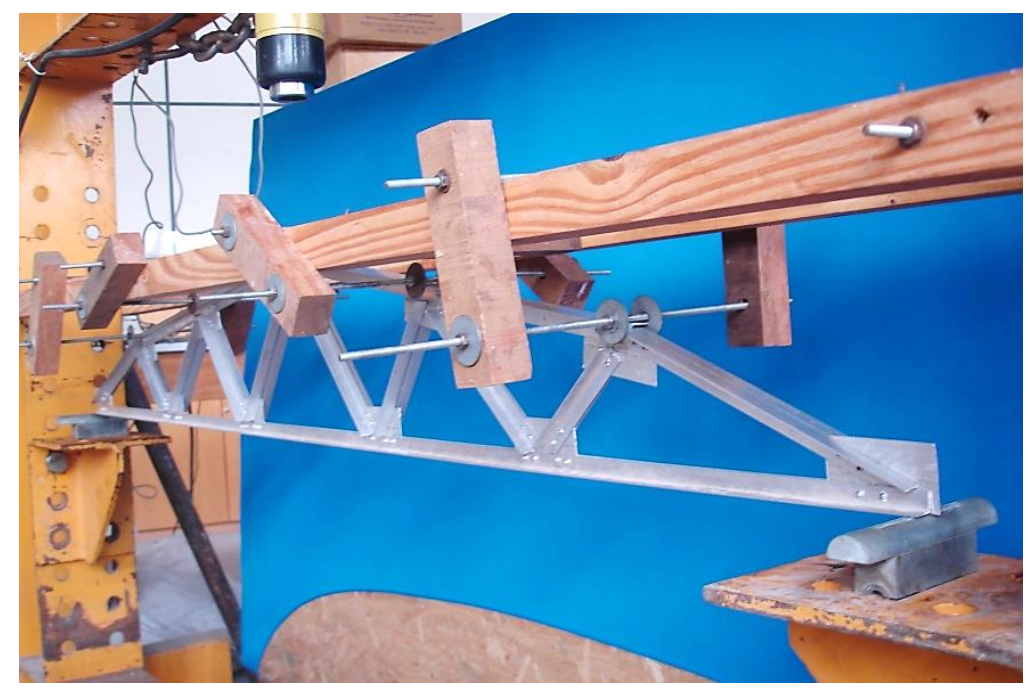

Figura 7: Modelo 2.

\begin{tabular}{|c|c|c|c|}
\hline \multicolumn{4}{|c|}{ TABELA 3: Resultados obtidos no ensaio. } \\
\hline Modelo & Peso próprio (KN) & Carga de colapso (KN) & Relação carga/peso próprio \\
\hline $\mathbf{1}$ & 0,01888 & 3,66 & 193,84 \\
\hline $\mathbf{2}$ & 0,01467 & 3,23 & 220,24 \\
\hline Dif (\%) & 22,33 & & \\
\cline { 1 - 2 } & & &
\end{tabular}

Da Tabela 3 observa-se que ambas as treliças resistiram ao carregamento de projeto $(3,2 \mathrm{KN})$, sendo que para o Modelo 2 a relação resistência/peso próprio foi ainda maior do que para o Modelo 1 (cerca 13,6 \%). Pode-se constatar que a significativa redução no peso da estrutura se deu sem o comprometimento da eficiência.

\section{CONCLUSÕES}

De forma geral, os resultados obtidos evidenciam a grande economia proporcionada pela aplicação de técnicas de otimização no projeto de estruturas. Nesse sentido, as treliças apresentam um campo bastante propício, uma vez que a redução no peso próprio pode ser obtida não apenas pela alteração nas dimensões das seções transversais dos elementos, mas também pela mudança na geometria e na topologia da estrutura. Dentro desse contexto, o Método do Recozimento Simulado, ou Simulated Annealing, apresenta um bom desempenho, ao permitir que se escape dos mínimos locais durante o processo de otimização.
A construção e o ensaio das treliças de alumínio permitiram a comprovação prática dos resultados numéricos, através da obtenção de estruturas que suportaram uma mesma carga de projeto, porém com decréscimo de 22,3\% no consumo de material na treliça para a qual se considerou um número maior de variáveis de projeto na otimização.

\section{REFERÊNCIAS BIBLIOGRÁFICAS}

KIRKPATRICK, S.; GELATT, C. D.; VECCHI M.P. Optimization by Simulated Annealing. Science, v. 220, n. 4598, p. 671-680, 1983.

KRIPKA, M. Discrete optimization of trusses by simulated annealing. J. Braz. Soc. Mech. Sci \& Eng., v. 26, n. 2, p. 170-173, abr./jun. 2004.

KRIPKA, M.; DREHMER, G. A. Determinação da geometria e configuração ótimas em treliças metálicas de banzos paralelos. In: PRAVIA, Zacarias $M$. Chamberlain; KRIPKA, Moacir. Construção Metálica: Estudos e Pesquisas Recentes. Passo Fundo: UPF Editora, 2005. p. 100-119. 
BUZINELLI, D. V. Projeto de elementos estruturais em alumínio. São Carlos, 2000. 160p. Dissertação (Mestrado) - Escola de Engenharia de São Carlos,Universidade de São Paulo.

ALCOA. Catálogo de Perfis Alcoa. Disponível em: http://www.alcoa.com/brasil/pt/info_page/downloads. asp. Acesso em: 23 set. 2013. 